\title{
The role of radioactive nickel in shaping the plateau phase of Type II supernovae
}

\author{
Alexandra Kozyreva, ${ }^{1,2 \star}$ Ehud Nakar, ${ }^{2}$ Roni Waldman ${ }^{3}$ \\ ${ }^{1}$ Max-Planck-Institut für Astrophysik, Garching bei München, 85748, Germany \\ ${ }^{2}$ The Sackler School of Physics and Astronomy, Tel Aviv University, Tel Aviv, 6997212, Israel \\ ${ }^{3}$ Racah Institute of Physics, The Hebrew University, Jerusalem 91904, Israel
}

Accepted XXX. Received YYY; in original form ZZZ

\begin{abstract}
In the present study, we systematically explore the effect of the radioactive ${ }^{56} \mathrm{Ni}$ and its mixing properties in the ejecta on the plateau of Type IIP supernovae ( $\mathrm{SNe}$ ). We evaluate the importance of ${ }^{56} \mathrm{Ni}$ in shaping light curves of SNe IIP by simulating light curves for two red supergiant models using different amounts of ${ }^{56} \mathrm{Ni}$ and with different types of mixing: uniform distribution of ${ }^{56} \mathrm{Ni}$ out to different fractions of the envelope and "boxcar" distribution of ${ }^{56} \mathrm{Ni}$. We find, similarly to previous studies, that ${ }^{56} \mathrm{Ni}$ extends duration of the plateau. We find a formula to estimate the extension based on the observed bolometric light curves and show that for most SNe IIP ${ }^{56} \mathrm{Ni}$ extends the plateau by about $20 \%$. Another effect of ${ }^{56} \mathrm{Ni}$ consists in reduction of the plateau decline rate, i.e. ${ }^{56} \mathrm{Ni}$ presented in the ejecta flattens the plateau. Our simulations suggest that for typical SNe IIP it can reduce the decline rate by about $1 \mathrm{mag} / 100$ day. We find that for the contribution of ${ }^{56} \mathrm{Ni}$ seen in most $\mathrm{SNe}$ our simulated bolometric light curves resemble observed ones for various types of ${ }^{56} \mathrm{Ni}$ mixing. We thereby cannot determine the level of ${ }^{56} \mathrm{Ni}$ mixing in these $\mathrm{SNe}$ based on the light curve alone. However, for SN 2009ib we find that only a model where ${ }^{56} \mathrm{Ni}$ is mixed significantly throughout most of the hydrogen envelope is consistent with the observed light curve. Our light curves are available via link https://wwwmpa.mpa-garching.mpg.de/ ccsnarchive/data/Kozyreva2018/.
\end{abstract}

Key words: supernovae: general - supernovae - stars: massive - radiative transfer

\section{INTRODUCTION}

Type II supernovae ( $\mathrm{SNe}$ II), i.e. those supernovae (SNe) which display strong hydrogen lines in the spectra at the time of discovery, are the most common explosions in the volume-limited sample (Smartt 2009; Smith et al. 2011; Li et al. 2011). Among them, Type IIP $\mathrm{SNe}$ remain bright during about 100 days showing the so-called plateau phase. They contribute $50 \%$ to $80 \%$ to all core-collapse explosions. Roughly $75 \%$ of all stellar explosions are core-collapse SNe (Mackey et al. 2003; Dahlen et al. 2004; Mannucci et al. 2007). CCSNe originate from explosions of massive stars, i.e. stars with initial masses above $8 \mathrm{M}_{\odot}$ and below $100 \mathrm{M}_{\odot}$. Progenitors for SNe II are stars which retain hydrogen envelopes by the time of iron-core collapse, among which extended red supergiants produce SNe IIP (Shklovskii 1960; Grasberg et al. 1971; Smartt 2009).

A given progenitor with a particular radius, mass, density, chemical structure, and explosion energy defines a unique light curve. However, the common task is to solve the reverse problem, i.e. determine the progenitor parameters from observational signa-

^ E-mail: sasha@mpa-garching.mpg.de tures of the SN explosion. Early studies estimated the progenitor and explosion parameters from the SN IIP light curve and photospheric velocity observations taking into account that the plateau phase is supported mostly by the thermal energy deposited by the shock wave which unbinds the progenitor envelope and that its evolution is dictated by the cooling and recombination wave receding through the expanding envelope (Grasberg et al. 1971; Grasberg \& Nadezhin 1976; Falk \& Arnett 1977; Litvinova \& Nadezhin 1985; Popov 1993). These studies provide formulae that relate light curve properties, mostly the plateau luminosity and duration and the photospheric velocity, to the most general progenitor properties (mass and radius) and the explosion energy. This is done using models with different levels of approximations of the the recombination wave that crosses the hydrogen envelope, neglecting contribution from freshly made radioactive ${ }^{56} \mathrm{Ni}$. However, more recent studies have shown that the energy input from radioactive decay of nickel ${ }^{56} \mathrm{Ni}$ and cobalt ${ }^{56} \mathrm{Co}$ strongly affects the behavior of the cooling wave and the resulting observations in SNe IIP (Young 2004; Kasen \& Woosley 2009; Bersten et al. 2011, and others). As a result, estimates which ignore the contribution of ${ }^{56} \mathrm{Ni}$ can be highly inaccurate. 
Table 1. Key characteristics of the input models.

\begin{tabular}{|c|c|c|c|c|c|c|c|c|c|c|c|c|}
\hline model & $\begin{array}{c}\text { Radius } \\
{\left[\mathrm{R}_{\odot}\right]} \\
\end{array}$ & $\begin{array}{l}\mathrm{M}_{\text {tot }} \\
{\left[\mathrm{M}_{\odot}\right]}\end{array}$ & $\begin{array}{c}\mathrm{M}_{\mathrm{H}} \\
{\left[\mathrm{M}_{\odot}\right]}\end{array}$ & $\begin{array}{c}\mathrm{M}_{\mathrm{He}} \\
{\left[\mathrm{M}_{\odot}\right]}\end{array}$ & $\begin{array}{c}\mathrm{M}_{\mathrm{C}} \\
{\left[\mathrm{M}_{\odot}\right]}\end{array}$ & $\begin{array}{c}\mathrm{M}_{\odot} \\
{\left[\mathrm{M}_{\odot}\right]} \\
\end{array}$ & $\begin{array}{l}\mathrm{M}_{\mathrm{Ne}} \\
{\left[\mathrm{M}_{\odot}\right]}\end{array}$ & $\begin{array}{c}\mathrm{M}_{\mathrm{Ni}} \\
{\left[\mathrm{M}_{\odot}\right]} \\
\end{array}$ & \multicolumn{2}{|c|}{$\begin{array}{c}\text { mixed in } \\
\text { [fraction of ejecta] }\end{array}$} & & $\begin{array}{c}E_{\text {expl }} \\
{\left[\text { foe }\left(\equiv 10^{51} \mathrm{erg}\right)\right]}\end{array}$ \\
\hline \multirow[t]{6}{*}{$\mathrm{m} 12$} & 496 & 11.25 & 5.4 & 3.8 & 0.09 & 0.6 & 0.04 & 0 & centre & uniform & & 0.4 \\
\hline & & & & & & & & 0.011 & $0.22 \mathrm{M}_{\odot}$ & $1 / 3$ & 1 & 0.9 \\
\hline & & & & & & & & 0.025 & & & & 1.35 \\
\hline & & & & & & & & 0.045 & & & & \\
\hline & & & & & & & & 0.065 & & & & \\
\hline & & & & & & & & 0.137 & & & & \\
\hline \multirow[t]{4}{*}{$\mathrm{m} 15$} & 631 & 13.4 & 6.0 & 4.3 & 0.17 & 0.2 & 0.24 & 0 & centre & boxcar & & 0.53 \\
\hline & & & & & & & & 0.028 & $0.4 \mathrm{M}_{\odot}$ & $0.16 \quad 0.31$ & & 1.1 \\
\hline & & & & & & & & 0.056 & & uniform & & 1.53 \\
\hline & & & & & & & & 0.113 & & 0.46 & & \\
\hline
\end{tabular}
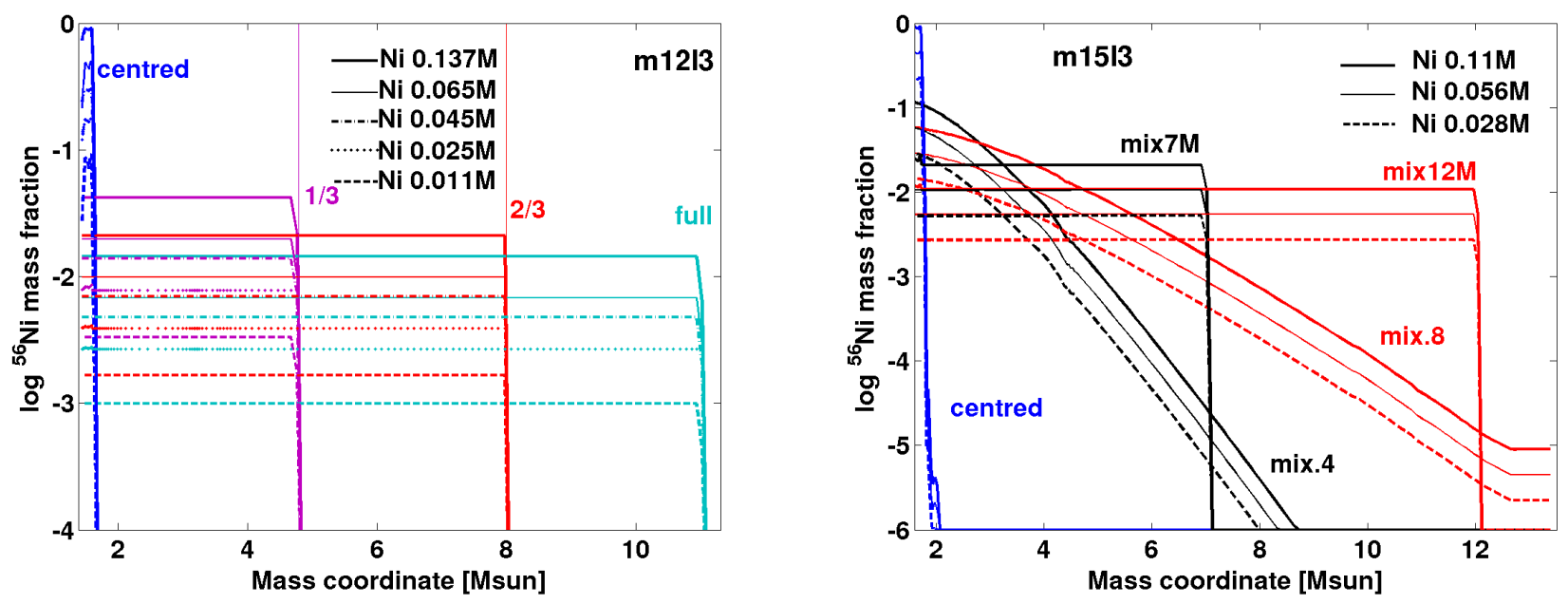

Figure 1. Distribution of ${ }^{56} \mathrm{Ni}$ in the ejecta of the model $\mathrm{m} 12$ (left) and of the model $\mathrm{m} 15$ (right). See explanation in the text.

The energy deposited by the radioactive decay of ${ }^{56} \mathrm{Ni}$ starts affecting the observed emission as soon as the recombination wave encounters Ni-generated photons diffusing through the inner ejecta. Once that happens ${ }^{56} \mathrm{Ni}$ energy deposition tends to increase the luminosity compared to the emission if no ${ }^{56} \mathrm{Ni}$ was present. Since ${ }^{56} \mathrm{Ni}$ contribution is more dominant at later times, it has two major effects. First, it delays the propagation of the recombination wave, thereby extending the plateau duration (e.g., Kasen \& Woosley 2009). Second, it reduces luminosity decline rate, making the plateau "flatter" (e.g., Bersten et al. 2011). The exact effect depends on the total abundance of ${ }^{56} \mathrm{Ni}$ and on its mixing throughout the envelope.

Recently, Nakar et al. (2016) introduced an observable that measures the importance of ${ }^{56} \mathrm{Ni}$ in the light curve of Type II SNe. They also analysed 24 observed SNe IIP and evaluated the importance of ${ }^{56} \mathrm{Ni}$ heating for the plateau phase. They concluded that ${ }^{56} \mathrm{Ni}$ contributes to most SN IIP plateaus in their study and plays an important role where the effect consists of both an extension and a flattening of the plateau. In Section 2 we give a brief description of the observable introduced by Nakar et al. (2016) and of the results of their analysis.

The goal of this paper is to study numerically the effect of ${ }^{56} \mathrm{Ni}$ on the light curve of type II SNe, and especially how the signature of ${ }^{56} \mathrm{Ni}$ depends on its mixing through the envelope. In order to do that we carry out simulations of two red supergiant progenitors and their explosions (Section 3). We explore the effect of Ni-heating during photospheric phase, by varying the amount of ${ }^{56} \mathrm{Ni}$ and its mixing as well as the explosion energy (Section 3.1), and analyse the obtained light curves in the context of observations using the measures introduced in Nakar et al. (2016) (Section 4). We summarise the conclusions of our study in Section 5.

\section{OBSERVABLE THAT MEASURES THE IMPORTANCE OF ${ }^{56} \mathbf{N i}$}

The main difficulty in measuring the effect of ${ }^{56} \mathrm{Ni}$ is that radiative transfer couples the energy deposited by the ${ }^{56} \mathrm{Ni}$ to the energy deposited by the SN shock (e.g., by affecting the ionization and thereby the opacity) in a way that the instantaneous luminosity cannot be separated to the contribution of each component. However, Nakar et al. (2016) have shown that there are integrated observable quantities where this separation is possible. They generalized the result of Katz et al. (2013) that have shown that the integral of the time weighted bolometric luminosity is a highly accurate measure of the integral over the time weighted energy deposition. Now, since the amount of ${ }^{56} \mathrm{Ni}$ in Type II SNe can be measured quite accurately 
from their nebular phase, one can separate the time weighted energy deposition to that of the initial energy deposited by the shock and the additional energy deposited by radioactive decay. Nakar et al. (2016), therefore, defined the observable:

$$
\eta_{\mathrm{Ni}}=\frac{\int_{0}^{t_{\mathrm{Ni}}} t Q_{\mathrm{Ni} 56} d t}{\int_{0}^{t_{\mathrm{Ni}}} t\left(L_{\mathrm{bol}}-Q_{\mathrm{Ni} 56}\right) d t},
$$

where $t$ is the time since the explosion, $L_{\mathrm{bol}}(t)$ is the instantaneous bolometric luminosity and $Q_{\mathrm{Ni} 56}(t)$ is the instantaneous deposition of energy by radioactive decay. $t_{\mathrm{Ni}}$ is the time that the photospheric phase ends, which is marked by the end of luminosity drop at the end of the plateau and the beginning of the the ${ }^{56} \mathrm{Co}$ tail.

The time weights in the integrals account for the adiabatic losses of the radiation between the time that the energy is deposited and the time that it is released, making this observable to be one of the few that are insensitive to the unknown details of the radiation transfer through the envelope. The numerator is insensitive to the radiation deposited by the $\mathrm{SN}$ shock. it measures the integrated time weighted luminosity that we would have seen if all the emission were powered by ${ }^{56} \mathrm{Ni}$ (as, for example, in Type I SNe) and it is roughly proportional to $M_{\mathrm{Ni} 56}$. The denominator is insensitive to the presence of ${ }^{56} \mathrm{Ni}$ and it measures the integrated time weighted luminosity that we would have seen if there were no ${ }^{56} \mathrm{Ni}$. Shussman et al. (2016) studied the physical meaning of the denominator, which they denote as ET. They show that it depends on the progenitor structure as well as the explosion energy and that for red-supergiants that retain most of their $\mathrm{H}$ envelope it can be roughly approximated as $E T \stackrel{\sim}{\sim} E^{1 / 2} M_{e j}^{1 / 2} R_{0}$, where $E$ is the kinetic energy at infinity (defined as the explosion energy), $M_{e j}$ is the ejecta mass and $R_{0}$ is the progenitor radius.

$\eta_{\mathrm{Ni}}$ is a measure of the importance of ${ }^{56} \mathrm{Ni}$ in shaping the emission that we see. If $\eta_{\mathrm{Ni}} \ll 1$ then ${ }^{56} \mathrm{Ni}$ is unimportant and there is a little difference between the observed light curve and the one that would have been observed if there were no ${ }^{56} \mathrm{Ni}$. If $\eta_{\mathrm{Ni}} \gg 1$ it implies that most of the observed emission is generated by ${ }^{56} \mathrm{Ni}$ (this is the case in type I SNe). Nakar et al. (2016) analysed 24 type II SNe with a good bolometric (or pseudo-bolometric) light curves and calculated the value of $\eta_{\mathrm{Ni}}$ for these SNe. They find that for all SNe except one, $\eta_{\mathrm{Ni}}$ falls within the range $0.1-0.7$. SN 2009ib is an exception which has $\eta_{\mathrm{Ni}}=2.6$.

${ }^{56} \mathrm{Ni}$ is expected to affect the decline rate during the plateau as well, and indeed Nakar et al. (2016) found that $\eta_{\mathrm{Ni}}$ is anti-correlated with the decline rate. ${ }^{56} \mathrm{Ni}$ is also supposed to extend the duration of the plateau. In the following section we examine these expectations using numerical simulations.

\section{INPUT MODELS}

For our analysis we computed two hydrogen-rich red supergiant models $\mathrm{m} 12$ and $\mathrm{m} 15$ with initial masses of $12 \mathrm{M}_{\odot}$ and $15 \mathrm{M}_{\odot}$, correspondingly (see Table 1). The models are at solar metallicity and non-rotating. The mixing-length parameter is chosen equal to 3 . The main property of the models is the presence of a hydrogenrich (total hydrogen mass $5.4 \mathrm{M}_{\odot}$ and $6 \mathrm{M}_{\odot}$ ) extended envelope $\left(496 \mathrm{R}_{\odot}\right.$ and $\left.631 \mathrm{R}_{\odot}\right)$. We apply the following method. Firstly, the stellar evolution from zero-age main sequence until the formation of an iron core was computed with MESA ${ }^{1}$. Secondly, the models were

1 Modules for Experiments in Stellar Astrophysics http://mesa. sourceforge.net/ (Paxton et al. 2011, 2013, 2015). blown up with V1D. Explosion is created by means of a piston, which is set at a Lagrangian mass of choice, given initial velocity equal to the escape velocity, and then allowed to free-fall. Thirdly, were mapped into the radiation hydrodynamics code STELLA to follow the post-explosion evolution. V1D is a one-dimensional hydrodynamics version of the code Vulcan (Livne 1993). V1D solves the equations of motion using explicit Lagrangian hydrodynamics. The radiative-transport in V1D is solved under the assumption of LTE and diffusion approximation for radiative transfer. The opacities in V1D are computed based on the opacity routines of CMFGEN (Dessart \& Hillier 2010; Dessart et al. 2010, 2015). Our main light curve simulations are carried out with STELLA which is a one-dimensional hydrodynamics code which solves radiative transfer equations in hundred frequency bins in momentum approximation (Blinnikov et al. 1998, 2006). Additionally, we carried out radiative transfer simulations with the multi-group extension to V1D and compare to the main results computed with STELLA.

The explosion of the $\mathrm{m} 12$ and m15 models by V1D was done using default explosion energy of 0.9 foe and 1.1 foe, correspondingly. To vary explosion energy, we modified velocity profile of the shocked material via multiplying by a certain factor while mapping the models into STELLA. The explosion energy of the models we run is defined as the kinetic energy at infinity and it is: 0.4 foe, 0.9 foe, and 1.35 foe for $\mathrm{m} 12$, and 0.53 foe, 1.1 foe, and 1.53 foe for $\mathrm{m} 15$.

\subsection{Nickel mixing setup}

In Figure 1, we demonstrate the input profiles for ${ }^{56} \mathrm{Ni}$ distribution in the ejecta. The values of ${ }^{56} \mathrm{Ni}$ mass that we set are, $0.011 \mathrm{M}_{\odot}$, $0.025 \mathrm{M}_{\odot}, 0.045 \mathrm{M}_{\odot}, 0.065 \mathrm{M}_{\odot}, 0.14 \mathrm{M}_{\odot}$, and no nickel for m12 (Fig. 1 left), and $0.028 \mathrm{M}_{\odot}, 0.056 \mathrm{M}_{\odot}, 0.11 \mathrm{M}_{\odot}$, and no nickel for $\mathrm{m} 15$ (Fig. 1 right). By default ${ }^{56} \mathrm{Ni}$ is concentrated to the inner $0.22 \mathrm{M}_{\odot}$ and $0.4 \mathrm{M}_{\odot}$ in the model $\mathrm{m} 12$ and the model $\mathrm{m} 15$, correspondingly. Default distribution of ${ }^{56} \mathrm{Ni}$ comes from V1D simulations of the piston-driven explosion. Nucleosynthesis in V1D is done with the implemented nuclear network which includes 54 isotopes. Reaction rates are as given in the non-smoker database https://nucastro.org/nonsmoker.html.

Throughout the paper we call this kind of unmixed distribution as "centrally located" or "centrally concentrated" ${ }^{56} \mathrm{Ni}$. We apply two kinds of mixing. For the model $\mathrm{m} 12$, we uniformly spread radioactive nickel in $1 / 3,2 / 3$ and in the entire (so-called "full") ejecta mass. For uniform mixing, ${ }^{56} \mathrm{Ni}$ is set as shown in Figure 1 while mass fraction of the rest species are recalibrated in each Lagrangian zone to keep the sum of mass fraction equal unity. For the model m15, we applied both uniform and so-called "boxcar" mixing. For boxcar mixing, we loop over all zones of the model. For each zone, with Lagrangian mass $m$, we uniformly mix the composition in all zones between $m$ and $m+d m$, where $d m$ is the boxcar parameter, e.g. $0.4,0.8$, in $\mathrm{M}_{\odot}$ units. We repeat the above procedure a total of four times.

The "boxcar" method is supposed to imitate mixing of chemical elements taking place during the earlier phase of expansion in corecollapse explosions. "Mix.4" means ${ }^{56} \mathrm{Ni}$ distribution in which we applied "boxcar"-mixing with the "boxcar"-parameter 0.4. "Mix.8" stands for mixing with the "boxcar"-parameter 0.8. In fact, $95 \%$ of ${ }^{56} \mathrm{Ni}$ is located in the inner $3.5 \mathrm{M}_{\odot}$ and $5.3 \mathrm{M}_{\odot}$ for "mix.4" and "mix.8", correspondingly, i.e. in 0.16 and 0.31 of the ejecta. "Mix7M" and "Mix12M" mean uniform mixing of ${ }^{56} \mathrm{Ni}$ in $7 \mathrm{M}_{\odot}$ $(46 \%)$ and $12 \mathrm{M}_{\odot}(88 \%)$ of the expanding ejecta, correspondingly. In total, each evolutionary model has 6 and 4 values for mass 
of ${ }^{56} \mathrm{Ni}, 3$ and 5 kinds of mixing, for $\mathrm{m} 12$ and $\mathrm{m} 15$ respectively, and 3 values for explosion energy.

\section{RESULTS}

\subsection{Light curves}

In Figures 2, 3 and 4, we present the resulting bolometric light curves for the model $\mathrm{m} 12$ and the model $\mathrm{m} 15$ with different amount of ${ }^{56} \mathrm{Ni}$ mass, degree of nickel mixing, and explosion energy. The labels along the curves indicate the explosion energy and corresponding average parameter $\eta_{\mathrm{Ni}}$. From these figures we can see several clear features.

First, the time at which ${ }^{56} \mathrm{Ni}$ starts to affect the light curve is determined only by the degree of mixing and is independent of the ${ }^{56} \mathrm{Ni}$ mass (see figure 4). A centrally concentrated ${ }^{56} \mathrm{Ni}$ starts affecting the emission around the time the plateau ends in the no ${ }^{56} \mathrm{Ni}$ light curve while a fully mixed ${ }^{56} \mathrm{Ni}$ has an affect already from the beginning of the plateau. In all cases, once ${ }^{56} \mathrm{Ni}$ starts affecting it increases the luminosity leading, as expected, to a flatter and longer plateau. The prominence of the ${ }^{56} \mathrm{Ni}$ emission is increased with the ${ }^{56} \mathrm{Ni}$ mass and reduced with the explosion energy. In general, for a given type of mixing, light curve with similar values of $\eta_{\mathrm{Ni}}$ (although different amounts of ${ }^{56} \mathrm{Ni}$ mass and explosion energy) show similar effect of ${ }^{56} \mathrm{Ni}$ on the light curve. The effect is of course more prominent for higher $\eta_{\mathrm{Ni}}$ values.

An interesting property of central ${ }^{56} \mathrm{Ni}$ mixing is that the light curve can be roughly separated between the cooling emission and the ${ }^{56} \mathrm{Ni}$ driven radiation. The luminosity of each phase depends on different properties of the progenitor, therefore, they are not necessarily similar. Indeed, in some of the light curves the transition between the two phases can be seen. The nature of this transition depends on $\eta_{\mathrm{Ni}}$. For very low values of $\eta_{\mathrm{Ni}} \lesssim 0.1$ the effect of ${ }^{56} \mathrm{Ni}$ can be hardly identified. For slightly higher values, but still relatively low, $\eta_{\mathrm{Ni}} \sim 0.2$, the ${ }^{56} \mathrm{Ni}$ contribution becomes apparent, but is still less luminous then earlier cooling emission, therefore, near the middle of the plateau, i.e. around day 50, the decay of the light curve becomes steeper. For intermediate values $\eta_{\mathrm{Ni}} \simeq 0.5$ the ${ }^{56} \mathrm{Ni}$ phase has comparable contribution as the cooling emission, and the transition between the two phases can be hardly observed. Finally, for $\eta_{\mathrm{Ni}} \gtrsim 1$ the ${ }^{56} \mathrm{Ni}$ driven emission is seen as a clear "bump" that starts rising from the middle of the plateau. Such a bump was never seen in a type II SN from a red supergiant. In simulations where ${ }^{56} \mathrm{Ni}$ is mixed out to the envelope its contribution is also mixed with that of the cooling emission leading to a smoother plateau evolution with no observable transition for any value of $\eta_{\mathrm{Ni}}$. A result of the difference between the mixing levels is that a more concentrated ${ }^{56} \mathrm{Ni}$ leads to longer plateaus than less concentrated ${ }^{56} \mathrm{Ni}$, while the flattening is more prominent when ${ }^{56} \mathrm{Ni}$ is mixed to outer layers. Light curves for the "boxcar" mixed models look similar to the models with the centrally located ${ }^{56} \mathrm{Ni}$ (for a given ${ }^{56} \mathrm{Ni}$ mass) during the plateau phase. This happens because the major fraction of ${ }^{56} \mathrm{Ni}$ in "boxcar" mixed models is located in the centre (see Section 3.1 and Table 1), and a little mass of ${ }^{56} \mathrm{Ni}$ is contained in the ejecta at higher velocity.

Interestingly, the values of $\eta_{\mathrm{Ni}}$ in most of the sample explored by Nakar et al. (2016) are in the range 0.3-0.7, which implies a non-negligible ${ }^{56} \mathrm{Ni}$ contribution. However, for these values it is hard to determine the type of ${ }^{56} \mathrm{Ni}$ mixing based on the light curve alone. The reason is that this is exactly the values were the ${ }^{56} \mathrm{Ni}$ phase in the models with centrally concentrated ${ }^{56} \mathrm{Ni}$ continues the cooling emission phase smoothly with no obvious observational feature. The only $\mathrm{SN}$ for which our results can strongly constrain the mixing is SN 2009ib for which $\eta_{\mathrm{Ni}}=2.6$. The light curve of SN 2009ib shows a long 130-day very smooth plateau (Takáts et al. 2015). This light curve is very different than those that we see in our simulations where the ${ }^{56} \mathrm{Ni}$ is concentrated in the centre. In fact, it seems that for $\eta_{\mathrm{Ni}}=2.6$ even in cases where ${ }^{56} \mathrm{Ni}$ is only partially mixed into the envelope we should identify the time at which the ${ }^{56} \mathrm{Ni}$ contribution kicks in. Among our light curves with similar $\eta_{\mathrm{Ni}}$ values only those with ${ }^{56} \mathrm{Ni}$ mixing throughout the envelope resemble the light curve of SN 2009ib.

We notice that bolometric light curves for some models have a specific step-like feature during transition from the standard recombination phase to the radioactive tail. These are models with either low explosion energy combined with low mass of ${ }^{56} \mathrm{Ni}$ located in the centre (see Figure 2, the model m12, $0.025 \mathrm{M}_{\odot}$ of ${ }^{56} \mathrm{Ni}, 0.4$ foe, blue solid curve) or models with "boxcar" mixing and low or medium explosion energy (see Figure 3, the model m15, e.g. $0.028 \mathrm{M}_{\odot}$, 0.53 foe and 1.1 foe, green and blue dashed curves). We noticed, that the step disappears for the mentioned models at higher explosion energy (e.g. with 1.53 foe in the model m15). In those models, the step is caused by helium recombination, i.e. when photosphere recedes to the helium shell. Photons produced in ${ }^{56} \mathrm{Ni}$ and ${ }^{56} \mathrm{Co}$ decay diffuse and ionise helium, therefore, keeping photosphere at this layer for a while. The step does not appear in cases of relatively higher energy, because the overall internal energy is higher and relative contribution from Ni-heating is lower. Similarly, the transition from hydrogen to helium recombination is smooth in the models with stronger Ni-mixing. In fact, in these cases $\mathrm{Ni}$-produced photons heat hydrogen-rich atmosphere equally as helium layer (in uniform mixing). While hydrogen ionisation supports photosphere at larger radius, the whole ejecta expands and then photosphere quickly drops through helium layer which becomes transparent. Nevertheless, the majority SNe IIP have no such shape in their light curves. Thus, our results suggest that all mentioned models which are attributed by the step-feature in the bolometric light curve are not the progenitors for normal SNe IIP. This result however is still needed to be confirmed by other numerical codes to verify that the observed step-like feature is indeed seen in these models. If confirmed then "boxcar" mixed models with low (about 0.5 foe) and intermediate ( 1 foe) explosion energy could not reproduce normal type IIP supernova light curves, while uniformly mixed models do.

As our study is focused on the light curve, we present ejecta properties on the coasting phase in Appendix A. Namely, we chose the model $\mathrm{m} 12$ with $0.045 \mathrm{M}_{\odot}$ of ${ }^{56} \mathrm{Ni}$ and the model $\mathrm{m} 15$ with $0.056 \mathrm{M}_{\odot}$ of ${ }^{56} \mathrm{Ni}$, and show selected species, hydrogen, helium, oxygen, silicon, and iron, presented in the $\mathrm{SN}$ ejecta along velocity.

\subsection{Extension of the plateau duration}

Our goal here is to quantify the effect of ${ }^{56} \mathrm{Ni}$ on the plateau duration $T_{p l}$, finding its dependence of the observable $\eta_{\mathrm{Ni}}$ and on the ${ }^{56} \mathrm{Ni}$ mixing. We measure $T_{p l}$ as time since explosion till the middle of transition between plateau and the radioactive tail. According to (Chugai 1991), duration of plateau corresponds to time when the recombination front traverses throught the $\mathrm{SN}$ ejecta. The plateau extension was previously studied. Kasen \& Woosley (2009) and Sukhbold et al. (2016) derived the following relation between the duration of the plateau, the explosion energy and the progenitor 

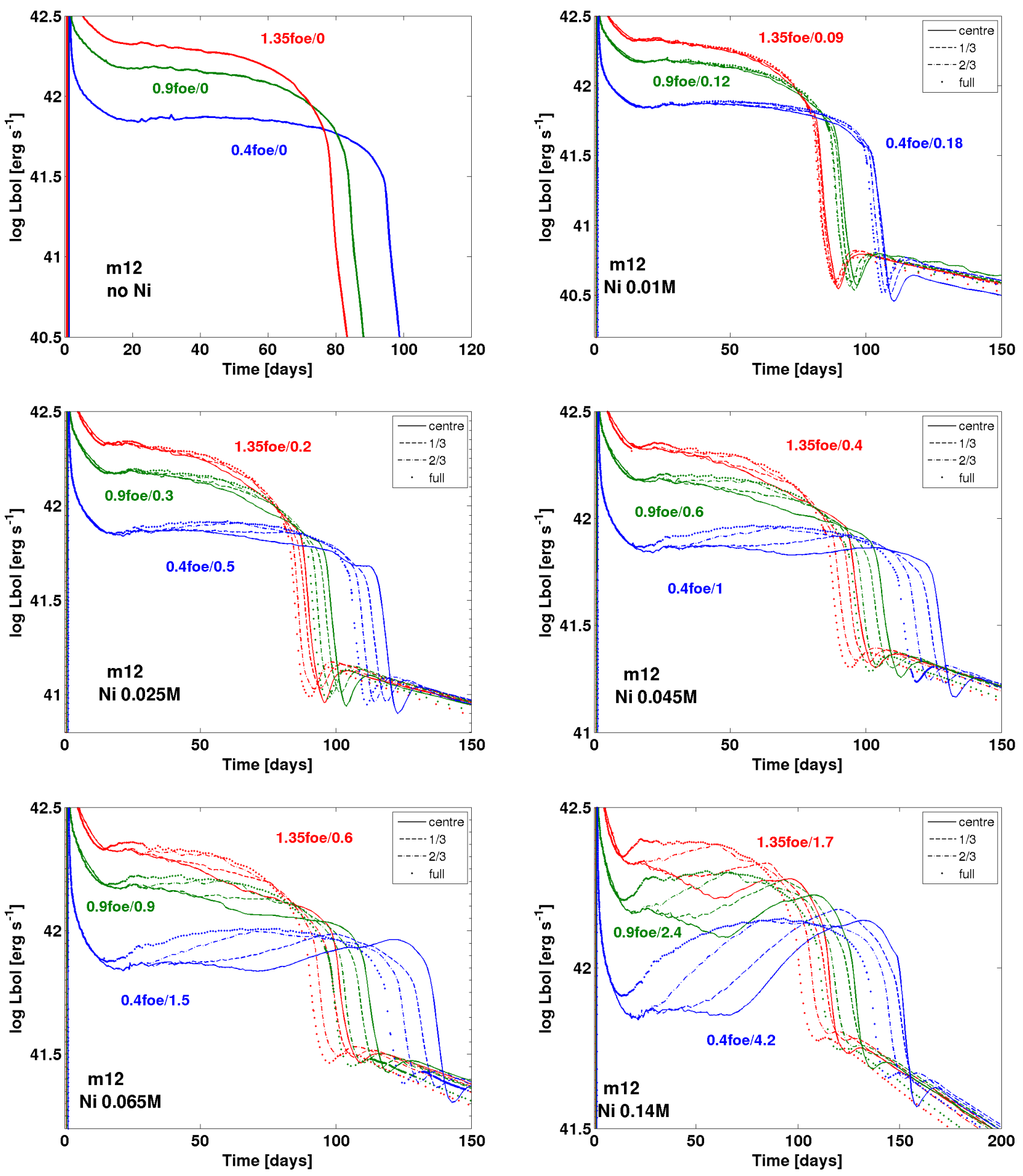

Figure 2. Bolometric light curves for the set of m15 models with different ${ }^{56} \mathrm{Ni}$ masses, different mixing of ${ }^{56} \mathrm{Ni}$, different explosion energies. See Table 1 for details. The labels indicate the explosion energy and $\eta_{\mathrm{Ni}}$.

properties based on a set of numerical runs (Eq. 19 of Sukhbold et al. (2016)):

$\frac{T_{p l}}{T_{p l}(N i=0)}=\left(1+C_{f} \frac{M_{\mathrm{Ni} 56}}{E_{51}^{1 / 2} M_{e j, 10}^{1 / 2} R_{0,500}}\right)^{1 / 6}$. where $C_{f}$ is a constant that depends on the progenitor structure, $E_{51}$ is the explosion energy in units of foe, $M_{e j, 10}$ is the ejecta mass is units of $10 \mathrm{M}_{\odot}$ and $R_{0,500}$ is the progenitor radius in units of $500 \mathrm{R}_{\odot}$. Interestingly, for a given progenitor structure $\eta_{\mathrm{Ni}} \propto$ 

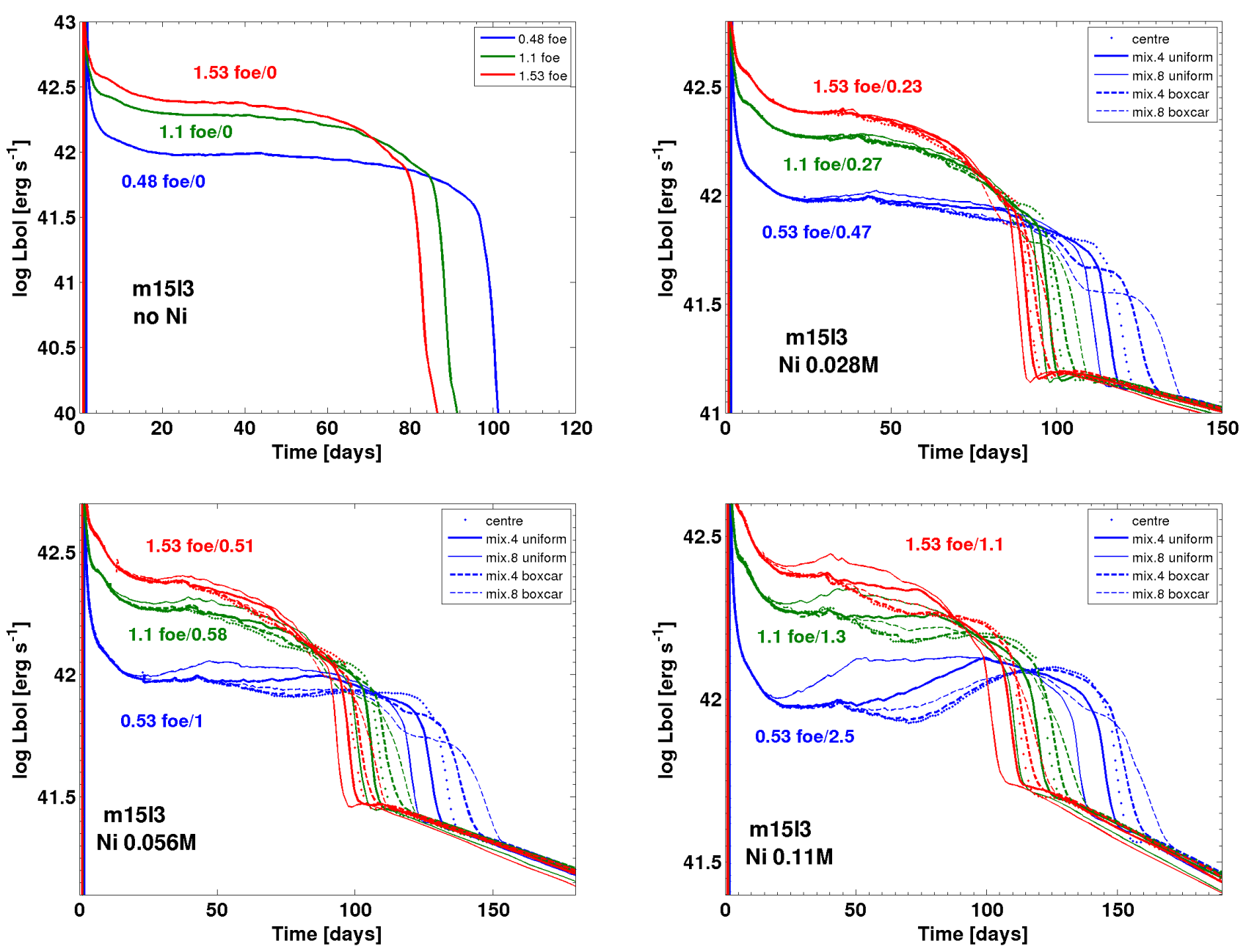

Figure 3. Bolometric light curves for the set of m15 models.

$\frac{M_{\mathrm{Ni} 56}}{E^{1 / 2} M_{e j}^{1 / 2} R_{0}}$ (see Section 2), therefore, we fit the results of our simulations to the relation

$\frac{T_{p l}}{T_{p l}(N i=0)}=\left(1+a \eta_{\mathrm{Ni}}\right)^{1 / 6}$.

The results are shown in Figure 5. We use different values of $a$ for different mixing types, where the range of $a$ values that we find is between 2 and 6 . Firstly, it is clear that formula 3 provides an excellent fit to the data. Its main advantage over equatio 2 is that $\eta_{\mathrm{Ni}}$ is an observable, therefore, it can be measured for any $\mathrm{SNe}$ with a good bolometric light curve. Secondly, it shows the dependence of the plateau extension on the mixing. Maximal extension is obtained for the boxcar "mix8" and "mix4" mixings followed closely by the centrally concentrated ${ }^{56} \mathrm{Ni}$. For these types of mixing $a \approx$ $5-6$. The plateau extension drops when ${ }^{56} \mathrm{Ni}$ is heavily mixed into the envelope, and the smallest effect is measured for fully mixed ${ }^{56} \mathrm{Ni}$ where $a \approx 2$. Finally, for our two progenitors we found that similar mixing types resulted in the same value of $a$. Although we examined only two progenitor models this suggests that most of the dependence of $C_{f}$ on the progenitor structure in equation 2 is absorbed into the parameter $\eta_{\mathrm{Ni}}$ and that the coefficient $a$ depends mostly on the mixing type. Hence, we suggest the averaged formula which is valid with roughly $10 \%$ accuracy:

$\frac{T_{p l}}{T_{p l}(N i=0)}=\left(1+4 \eta_{\mathrm{Ni}}\right)^{1 / 6}$.

Applying these results to the sample of Nakar et al. (2016) we find that for typical explosions with $\eta_{\mathrm{Ni}} \approx 0.5$ the ${ }^{56} \mathrm{Ni}$ extends the plateau by $15 \%-25 \%$, depending on the type of mixing. As the typical observed plateau duration is about 100 days, this implies that if there was no ${ }^{56} \mathrm{Ni}$ the typical plateau duration would have been about 80 days. In the case of SN 2009ib where the plateau is unusually long, we find that for $\eta_{\mathrm{Ni}}=2.6$ and a uniform mixing throughout the envelope (as inferred from the light curve shape) the ${ }^{56} \mathrm{Ni}$ extends the plateau by $35 \%$. Thus, the unusual plateau length of SN 2009ib is mostly due to ${ }^{56} \mathrm{Ni}$, as without ${ }^{56} \mathrm{Ni}$ the plateau duration would have been shorter than 100 days.

\subsection{Mitigating the plateau decline rate}

Another effect of ${ }^{56} \mathrm{Ni}$, when it is present in the SN ejecta, is increasing the plateau luminosity at late time, thereby reducing the decline rate, i.e., making the light curve flatter. We use the bolometric increase in magnitude (i.e., drop in luminosity) between days 25 and $75, \Delta m_{25-75}$, as a measure of the decline rate. The values 

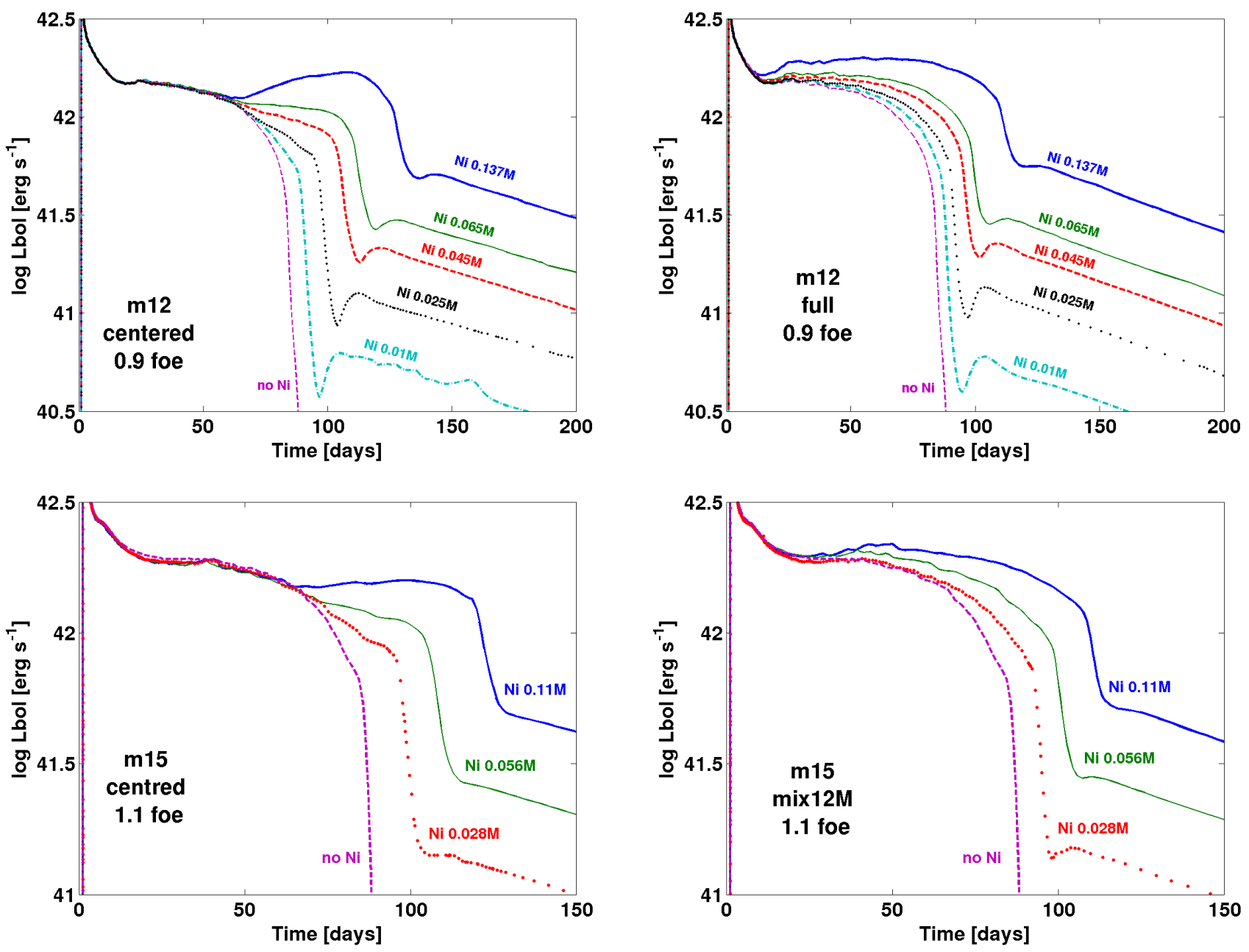

Figure 4. Bolometric light curves (same as in figures 2 and 3) where in each panel the amont of ${ }^{56} \mathrm{Ni}$ varies while the mixing and the energy reamins constant.

in the Nakar et al. (2016) sample vary between $\Delta m_{25-75}=-0.1$ for light curves that show slow brightening during the plateau to $\Delta m_{25-75}=1.2$ (i.e., a decline rate of $2.4 \mathrm{mag} / 100$ day) for fast declining $\mathrm{SN}$, which are usually classified as type IIL. Nakar et al. (2016) test for a correlation between $\eta_{\mathrm{Ni}}$ and $\Delta m_{25-75}$ finding a significant anti-correlation, suggesting that ${ }^{56} \mathrm{Ni}$ is responsible for at least some of the plateau flatness. They continue with an attempt to measure this effect, estimating that in $\mathrm{SNe}$ with flat plateaus ${ }^{56} \mathrm{Ni}$ contributes about $1 \mathrm{mag} / 100$ day to the plateau (i.e., without ${ }^{56} \mathrm{Ni}$ these $\mathrm{SNe}$ would have showen a decline rate of $\Delta m_{25-75} \approx 0.5$ ).

Figure 6 shows $\Delta m_{25-75}$ as a function of $\eta_{\mathrm{Ni}}$ for progenitor $\mathrm{m} 12$ (the results for $\mathrm{m} 15$ are very similar). The different panels are for different explosion energies. First, we find that without ${ }^{56} \mathrm{Ni}$ more energetic and luminous explosions evolve faster and have a faster decline rate. This is consistent with the correlation found between SN luminosity and the decline rate (Anderson et al. 2014; Faran et al. 2014b), suggesting that high explosion energy might be at least one of the reasons for the fast decline observed in some SNe IIL. The effect of ${ }^{56} \mathrm{Ni}$ on the decline rate is also seen clearly. For all mixing types higher ${ }^{56} \mathrm{Ni}$ mass results in slower decline rates. This effect is seen for all mixing types, although it is less prominent when ${ }^{56} \mathrm{Ni}$ is concentrated in the center. Quantitatively, for $\eta_{\mathrm{Ni}} \approx 0.5$ the effect on low energy explosion is minor, but on explosions with energy of about 1 foe it reduces the decline rate by about $1 \mathrm{mag} / 100$ days (i.e., reducing $\Delta m_{25-75}$ by 0.5 ). For $\eta_{\mathrm{Ni}}=0.1$, the effect is minor, and the resulting light curve decay is similar to the light curve without ${ }^{56} \mathrm{Ni}$. For $\eta_{\mathrm{Ni}}>1$, the plateau is always very flat, which is consistent with the light curve of SN 2009ib, and in some cases even slowly rising.

\subsection{Correlations between $\eta_{\mathrm{Ni}}$ and the drop from the plateau to the radioactive tail}

Figure 7 shows the drop in the bolometric light curve during the transition from the plateau to the tail as a function of $\eta_{\mathrm{Ni}}$. We define the transition as a difference in bolometric magnitude between the end of plateau (when the plateau slope starts changing noticeably) and the beginning of radioactive tail. It shows a rather tight correlation with $\eta_{\mathrm{Ni}}$ while the scatter is mostly due to the different mixing types. The transition between the plateau and the tail is a complicated characteristic which depends on the explosion energy, global progenitor properties and the mass of ${ }^{56} \mathrm{Ni}$ and its distribution, i.e. $\eta_{\mathrm{Ni}}$. 


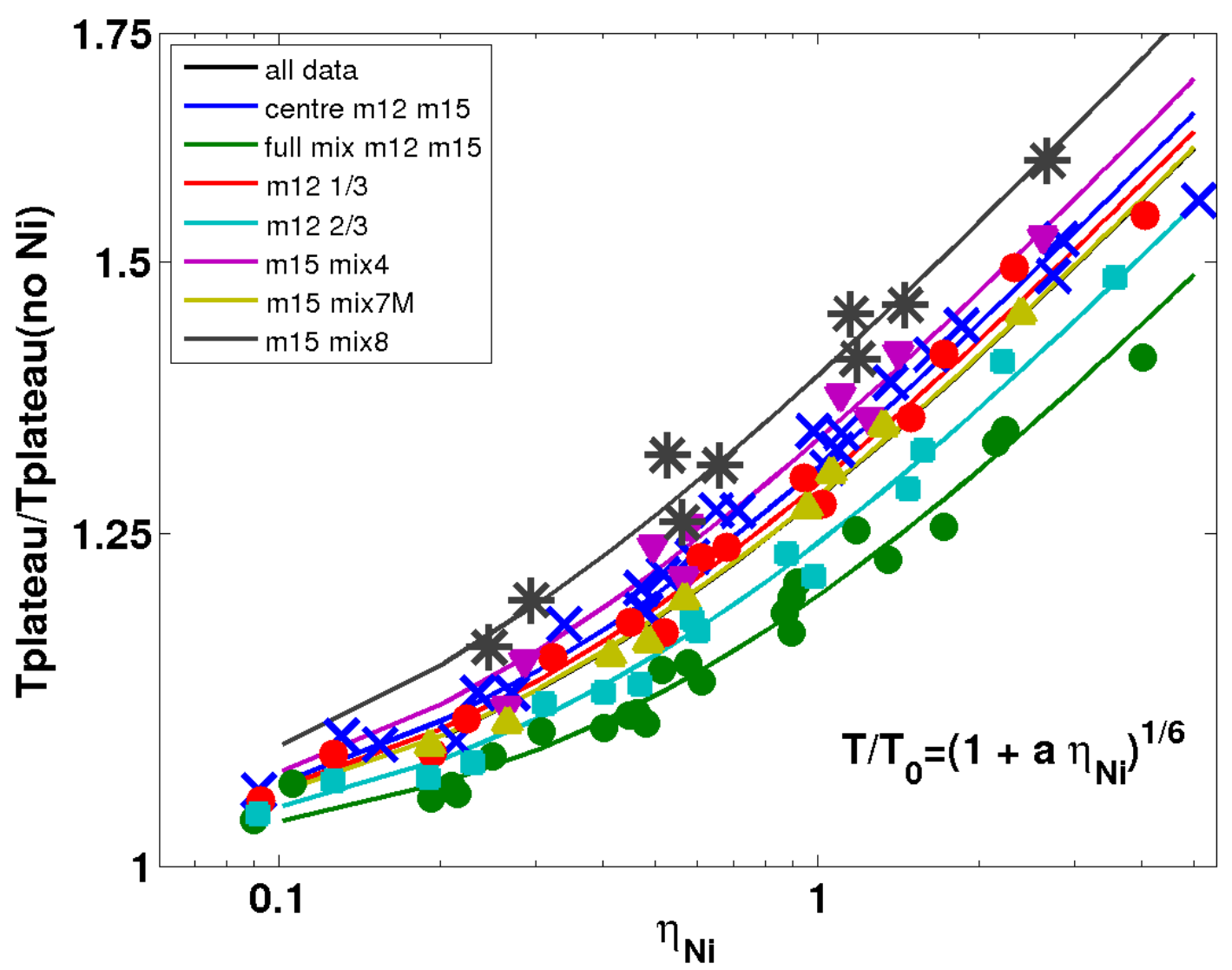

Figure 5. Plateau duration for models with ${ }^{56} \mathrm{Ni}$ relative to the models without ${ }^{56} \mathrm{Ni}$ along parameter $\eta_{\mathrm{Ni}}$.
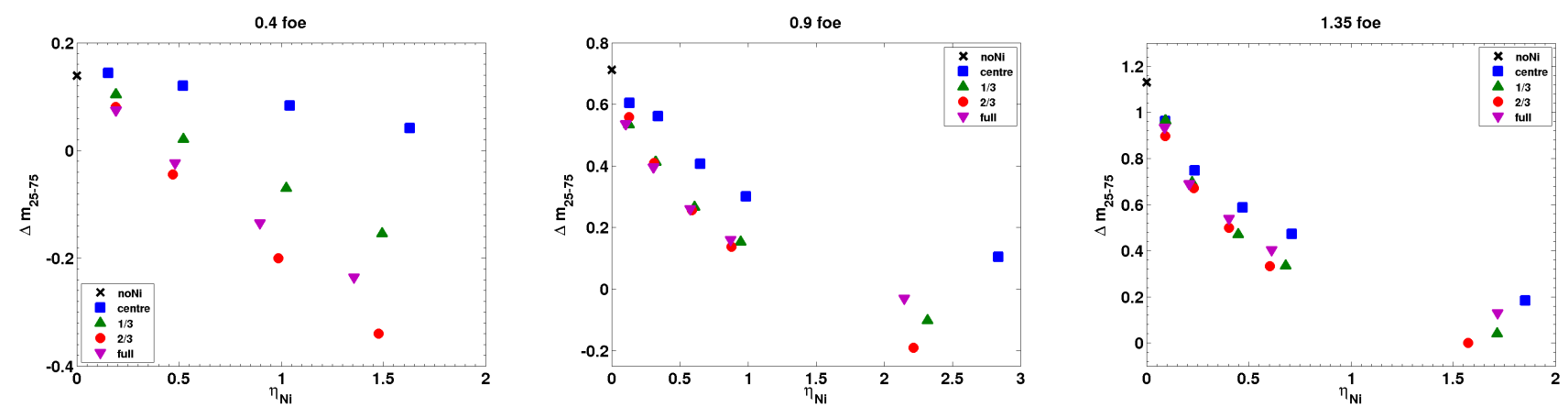

Figure 6. The effect of ${ }^{56} \mathrm{Ni}$ on the decline rate of explosions with different energies for diffrent mixing types.

\subsection{Photospheric velocity}

Theoretically, we expect that ${ }^{56} \mathrm{Ni}$ presented in the supernova ejecta heats and ionises matter while supplying high energy photons and positrons, therefore, keeping the photosphere at larger radii where velocity is higher. We define photospheric velocity as velocity of the Lagrangian zone where the integrated Rosseland optical depth is equal 2/3. In Figure 8, we depicts the evolution of the photospheric velocity models $\mathrm{m} 15$ with $0.065 \mathrm{M}_{\odot}$ of ${ }^{56} \mathrm{Ni}$ and different explosion energies, 0.53 foe, 1.1 foe, and 1.53 foe. It shows that the main factor that determines the photospheric velocity is the explosion energy. Since the typical ejecta velocity scales as $\sqrt{E / M}$, we expect the ejecta mass to have a similar effect as the explosion energy (e.g., Popov 1993). The effect of ${ }^{56} \mathrm{Ni}$ on the photospheric velocity is significant only at late times. Similarly to the light curve, higher level of mixing starts affecting the velocity at earlier time. However, this happens later than in the light curves. At day 50, only full mixing shows some ${ }^{56} \mathrm{Ni}$ effects of order $10 \%$. Towards the end of the plateau all mixing types affects the velocity, where in the case of uniform mixing the line velocity can be more than twice the velocity at the same time without ${ }^{56} \mathrm{Ni}$. To conclude, ${ }^{56} \mathrm{Ni}$ has a negligible contribution to the photospheric velocity evolution up to the middle of the plateau, and starts playing some role by the 


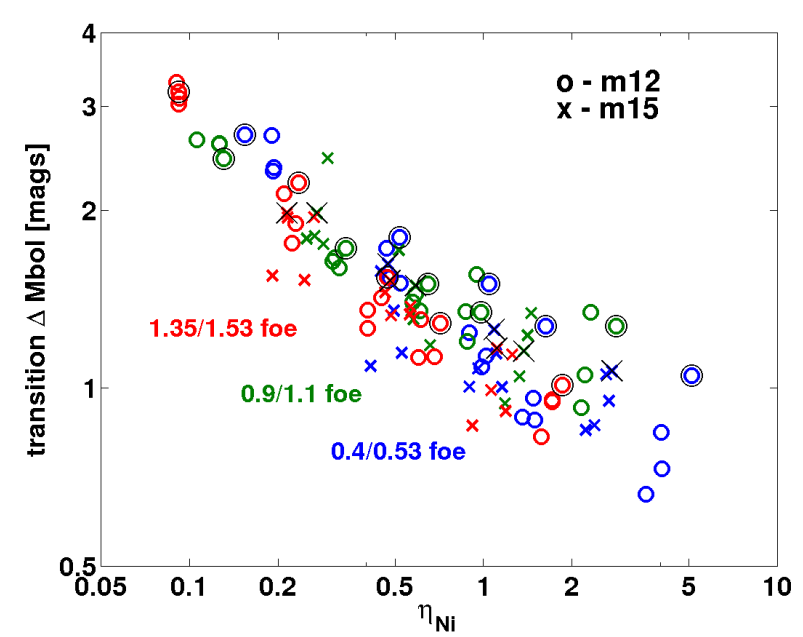

Figure 7. Correlation between drop of the bolometric light curve during transition from plateau and tail and $\eta_{\mathrm{Ni}}$. We marked central cases with additional black light circles and crosses for the models $\mathrm{m} 12$ and $\mathrm{m} 15$, respectively.

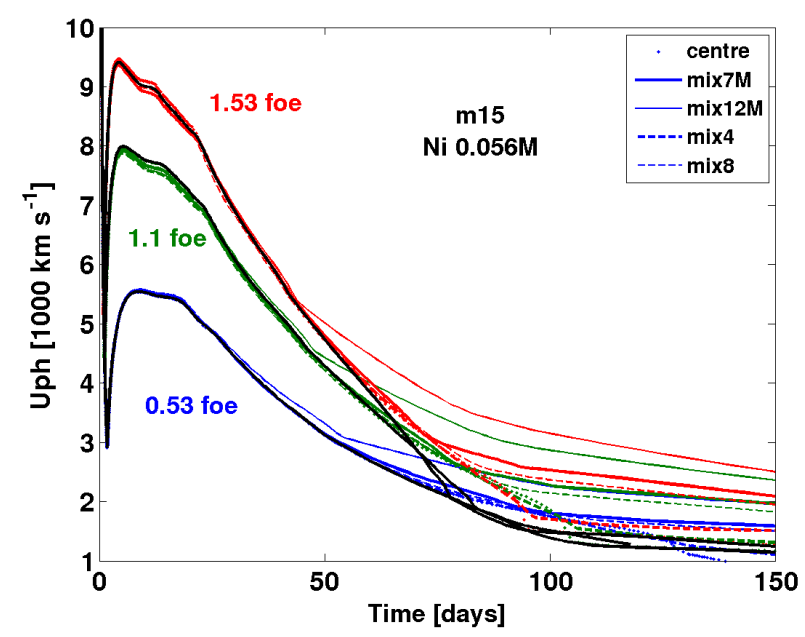

Figure 8. Photospheric velocity evolution for the model $\mathrm{m} 15$ with $0.065 \mathrm{M}_{\odot}$ of ${ }^{56} \mathrm{Ni}$ and different explosion energies, 0.53 foe (blue), 1.1 foe (green), and 1.53 foe (red). Black curves represent photospheric velocity evolution for the model $\mathrm{m} 15$ with no ${ }^{56} \mathrm{Ni}$ included.

end of plateau. However, it is expected that even small changes in photospheric velocity due to $\mathrm{Ni}$-heating will be seen in spectra.

Since the velocity at day 50 is often used to characterise the explosion energy and ejecta mass, we present the photospheric velocity at day 50 in Figure 9, for all models in the study along the parameter $\eta_{\mathrm{Ni}}$. We show that there is no major effect, and for $\eta_{\mathrm{Ni}} \approx 0.5$ the photospheric velocity is at most $10 \%$ faster.

\subsection{Colour $B-V$}

In Figure 10, we plot $B-V$ colour for model m15 with $0.056 \mathrm{M}_{\odot}$ of ${ }^{56} \mathrm{Ni}$ with various mixing types and explosion energies of 0.53 foe ("LowE", thick solid), 1.1 foe ("MediumE", thin solid), and 1.53 foe ("HighE", thick dashed). We compare our theoretical curves with a few normal SNe IIP: 1999em (crosses), 1999gi

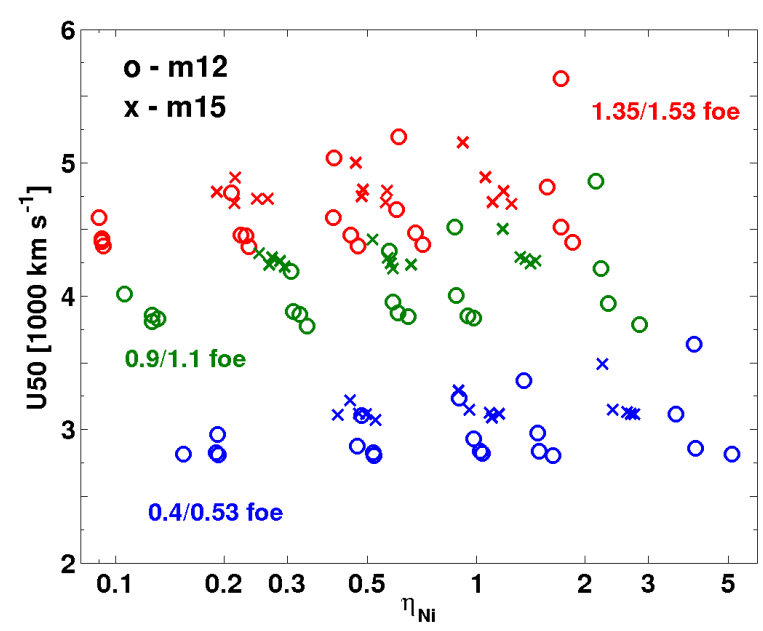

Figure 9. Photospheric velocity at day 50 for all models in the study.

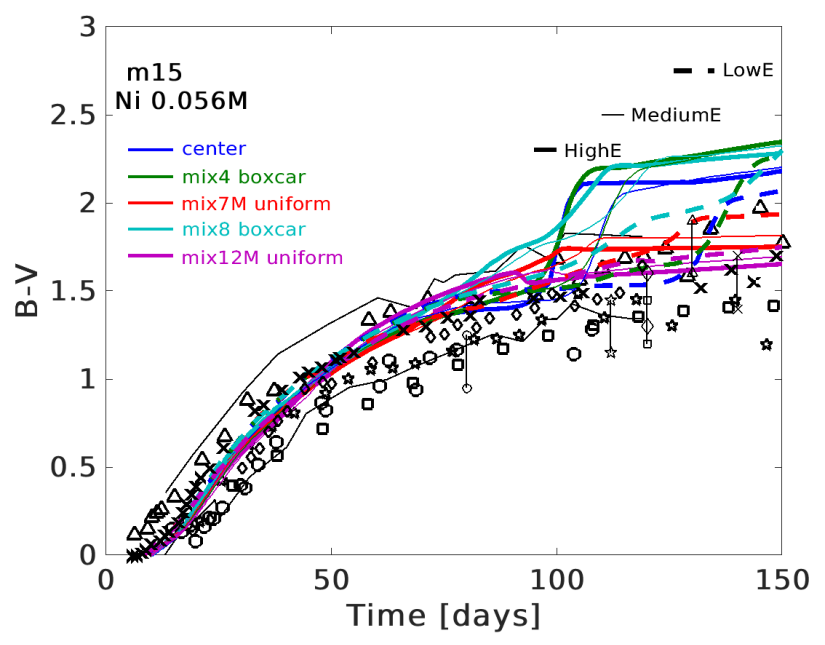

Figure 10. $B-V$ colour for the model $\mathrm{m} 15$ with $0.056 \mathrm{M}_{\odot}$ of ${ }^{56} \mathrm{Ni}$ mixed differently and different explosion energy, 0.53 foe ("LowE", thick solid), 1.1 foe ("MediumE", thin solid), and 1.53 foe ("HighE", thick dashed). Superposed symbols are for SNe IIP from Faran et al. (2014b,a) and Valenti et al. (2016). Among others, crosses stand for 1999em, triangles stand for 1999gi, stars stand for 2001X, diamonds stand for 2005ay, circles stand for 2013fs, and squares stand for ASASSN14ha. Vertical lines indicate the end of plateau phase in each individual SNe with symbols corresponding to the colour data. Vertical bars indicate the end of plateau for given SN. Symbols at the end of a bar correspond to the marker type of the curve.

(triangles), 2001X (stars), 2005ay (diamonds), 2013fs (circles), ASASSN14ha (squares) (Faran et al. 2014b; Valenti et al. 2016), and averaged $B-V$-curves (thin black curves, Faran et al. 2014b). We find that models with centrally concentrated ${ }^{56} \mathrm{Ni}$ (blue curves) and "boxcar" mixing (green and cyan curves) quickly change their $B-V$ colour during the transition from the plateau to the radioactive tail. This is in contrast to the observations that demonstrate monotonic evolution of $B-V$ colour. All the models with uniformly mixed ${ }^{56} \mathrm{Ni}$ show evolution that is similar to the observed one.

Our results suggest that the ${ }^{56} \mathrm{Ni}$ is well mixed into the envelope in normal SNe IIP. Note however, that STELLA produces reliable 
evolution of radiation field for supernova ejecta when a large fraction of the ejecta is optically thick, i.e. during photospheric phase. By the end of transition from the plateau to the tail, the ejecta become optically thin and the photosphere recedes deep into the ejecta. At this time the overall bolometric light curves predicted by STELLA are also reliable. However, lines begin playing a significant role at late time, and STELLA colours are less reliable. Therefore, detailed simulations with non-thermal effects and larger atomic data base (like it is done in CMFGEN, Li et al. (2012)) are needed to confirm our result about the $B-V$ colour evolution. Previously, Dessart \& Hillier (2011) present non-LTE simulations for two models. Their $B-V$ colour curves lie below the observed range marked in our Figure 10. Nevertheless, their light curves are not properly reproduce normal SNe IIP. Kasen \& Woosley (2009) present broad band light curves for a particular model with applied boxcar mixing. The derived $B-V$ colour is in very good agreement with our results for the "mix4" and "mix 8 " boxcar curves, i.e. the colour reddens in a step-like way during the transition to the radioactive tail. Note though that Kasen \& Woosley (2009) do not include non-LTE effects in their study.

\subsection{Comparison to V1D}

The opacity treatment remains the core aspect in the radiative transfer simulations which provide an uncertainty in the resulting data. Figure 11 demonstrates that using different sets of lines and different assumptions about thermodynamical equilibrium (LTE versus non-LTE) leads to visible differences in bolometric light curves.

In this section we compare simulations done with STELLA and V1D. V1D uses opacity tables compiled from the CMFGEN data (Dessart \& Hillier 2010; Dessart et al. 2010, 2015) which is significantly broader than the STELLA standard settings. CMFGEN includes about 500,000 lines and level populations without assumption of LTE, and treats non-thermal excitation, while STELLA has 160,000 lines and computed level populations based on modified Saha equations. From the comparison plots in Figure 11, it is obvious that the effective V1D opacity is larger than the opacity in STELLA. This makes the V1D plateau 7-day longer even in the model without radioactive nickel ${ }^{56} \mathrm{Ni}$ (upper panel of Figure 11). Apart from the plateau duration, luminosity on the plateau varies. Hence, V1D predicts a 0.04 dex dimmer plateau compared to STELLA in the case of $0.056 \mathrm{M}_{\odot}$ of ${ }^{56} \mathrm{Ni}$ (bottom panel of Figure 11). Nevertheless, the qualitative agreement between V1D and STELLA is very good.

Photospheric velocity estimated by V1D and STELLA is different, as seen in Figure 11, while photospheric temperature and radius are in very good agreement. V1D overestimates the velocity by $2000 \mathrm{~km} \mathrm{~s}^{-1}$ during the post-SBO cooling phase and up to the middle of plateau, and by $1000 \mathrm{~km} \mathrm{~s}^{-1}$ after the middle point. We explain this difference by the way V1D solves the radiative transfer equations, i.e. diffusion approximation.

\section{CONCLUSIONS}

In the present study, we carried out a systematic analysis of the impact of ${ }^{56} \mathrm{Ni}$ presented in the SN ejecta on the resulting light curves of SNe IIP. For this, we used two red supergiant models computed with MESA. We vary amount of ${ }^{56} \mathrm{Ni}\left(0.01-0.14 \mathrm{M}_{\odot}\right)$ and its mixing (centrally concentrated to full), and explosion energy ( 0.4 foe to 1.53 foe) and computed a set of light curves with STELLA. Our light curves are available via link https://wwwmpa.mpa-garching. mpg.de/ccsnarchive/data/Kozyreva2018/.

Based on our light curve simulations, we conclude that even
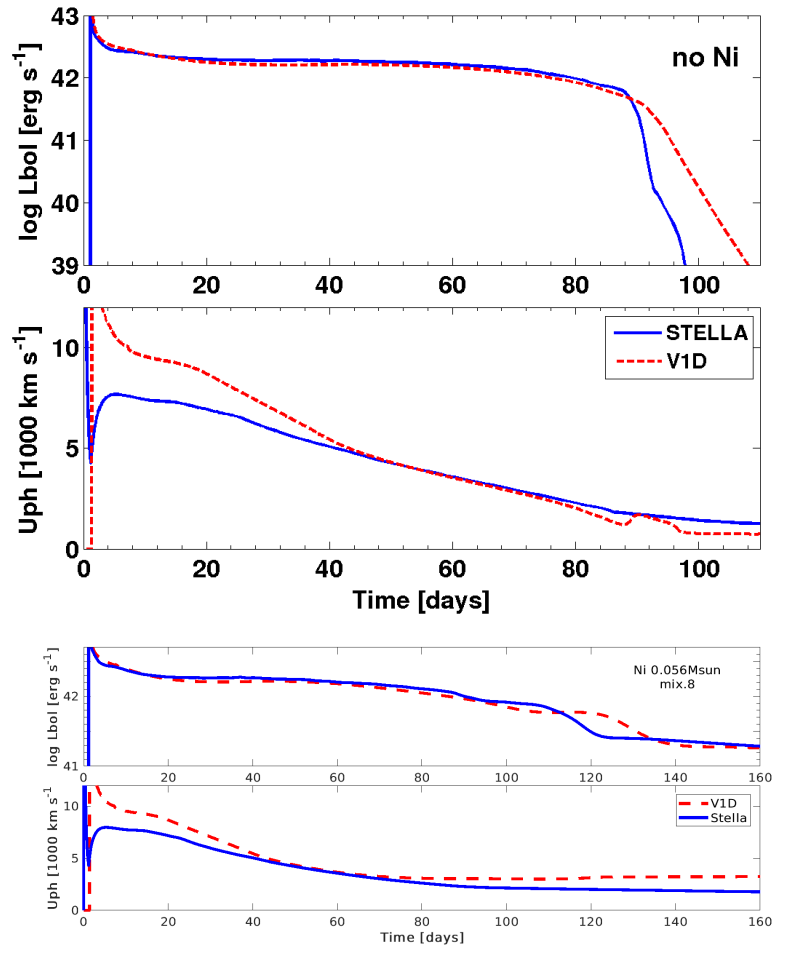

Figure 11. V1D (red dashed) and STELLA (blue solid) bolometric light curves and photospheric velocity for the model $\mathrm{m} 15$ with no radioactive material included (top) and with $0.056 \mathrm{M}_{\odot}$ if ${ }^{56} \mathrm{Ni}$ mixed in $80 \%$ of ejecta (bottom).

small amount of radioactive nickel ${ }^{56} \mathrm{Ni}$ presented in the supernova ejecta noticeably modifies the bolometric light curve. There is a combination of two effects from $\mathrm{Ni}$-heating on the plateau duration and shape: (1) radioactive nickel ${ }^{56} \mathrm{Ni}$ extends the plateau, and (2) ${ }^{56} \mathrm{Ni}$ flattens plateau decline rate.

To evaluate the importance of ${ }^{56} \mathrm{Ni}$ impact, we used the parameter $\eta_{\mathrm{Ni}}$ which is a ratio between time weighted ${ }^{56} \mathrm{Ni}$ deposited energy and the weighted shock deposited energy. We found that the extension of the plateau due to presence of ${ }^{56} \mathrm{Ni}$ can be accurately approximated by a simple formula with an argument $\eta_{\mathrm{Ni}}$ (Equation 3 and Figure 5). We found that in most observed type IIP SNe the plateau is extended by $15-25 \%$. We also found that ${ }^{56} \mathrm{Ni}$ effectively flattens the plateau decline. In fact, the drop in light curves between day 25 and day 75 is correlated with $\eta_{\mathrm{Ni}}$, i.e. decline rate is lower (a light curve is flatter) for higher $\eta_{\mathrm{Ni}}$. For an intermediate explosion energy of about 1 foe and $\eta_{\mathrm{Ni}} \approx 0.5$ the decline rate is reduced by about $1 \mathrm{mags} / 100$ days $\left(\Delta m_{25-75}=0.5 \mathrm{mags}\right.$ for $\eta_{\mathrm{Ni}}=0.5$, Figure 6) The common values of $\eta_{\mathrm{Ni}}$ for the observed SNe IIP is $0.3-0.7$ (Nakar et al. 2016), therefore, ${ }^{56} \mathrm{Ni}$ significantly contributes to plateau shape and duration.

Among other findings are:

- Regardless explosion energy and the total amount of radioactive ${ }^{56} \mathrm{Ni},{ }^{56} \mathrm{Ni}$ starts to affect plateau luminosity at particular time according to degree of mixing. Particularly, centrally located ${ }^{56} \mathrm{Ni}$ modifies the light curve at the end of the plateau, around day 75 , while fully mixed ${ }^{56} \mathrm{Ni}$ increases plateau luminosity at the most beginning, around day 20 . In all cases this time depends only on the extent of ${ }^{56} \mathrm{Ni}$ distribution (see Figure 2).

- For the typical values of $\eta_{\mathrm{Ni}}$ (between 0.3 and 0.7 ), it is difficult 
to distinguish contribution from pure recombination and cooling, and ${ }^{56} \mathrm{Ni}$ heating. Light curves alone do not provide enough information to differenciate between different degrees of mixing. The observed SN 2009ib with the long 150-day plateau has $\eta_{\mathrm{Ni}}=2.6$ and requires moderate amount of ${ }^{56} \mathrm{Ni}\left(0.046 \mathrm{M}_{\odot}\right)$ mixed heavily throughout the ejecta.

- "Boxcar" mixing of ${ }^{56} \mathrm{Ni}$ leads to a bolometric light curve with a double step transition from the plateau to the tail if explosion energy is relatively low, about 0.5 foe. This kind of feature is not observed in normal SNe IIP. However, additional numerical simulations are required to clarify this conclusion.

- A centrally concentrated ${ }^{56} \mathrm{Ni}$ and "boxcar" lead to a sharp jump in $B-V$ following the end of the plateau, which is not seen in observations. However, this aspect requires additional simulations accounting for non-LTE radiative transport.

- There is no significant modification to photospheric velocity up to day 50 due to ${ }^{56} \mathrm{Ni}$-heating. However, moderate changes occur later.

We highlight that uniformly mixed ${ }^{56} \mathrm{Ni}$ either in half of the ejecta or almost entire ejecta supports plateau luminosity providing light curves consistent with observetions. "Boxcar" and centrally concentrated ${ }^{56} \mathrm{Ni}$ results in light curves which colour evolution is inconsistent with observations. However, this should be confirmed by additional numerical simulations. If confirmed, this challenges the core-collapse explosion simulations, since conventional "boxcar" ${ }^{56} \mathrm{Ni}$ mixing is believed to mimic the realistic macroscopic mixing in the supernova ejecta and which is predicted by corecollapse simulations (Wongwathanarat et al. 2015; Müller et al. 2017; Utrobin et al. 2017).

\section{ACKNOWLEDGMENTS}

AK and EN are supported by ERC grant No. 279368 ("The Gamma Ray Burst - Supernova Connection and shock breakout physics") and partially by the I-Core center of excellence of the CHE-ISF. The STELLA simulations were carried out on the DIRAC Complexity system (grants ST/K000373/1 and ST/M006948/1), operated by the University of Leicester IT Services, which forms part of the STFC DiRAC HPC Facility (www . dirac . ac . uk). AK thank Sergey Blinnikov and Viktor Utrobin for fruitful and useful discussions.

\section{REFERENCES}

Anderson J. P., et al., 2014, ApJ, 786, 67

Bersten M. C., Benvenuto O., Hamuy M., 2011, ApJ, 729, 61

Blinnikov S. I., Eastman R., Bartunov O. S., Popolitov V. A., Woosley S. E., 1998, ApJ, 496, 454

Blinnikov S. I., Röpke F. K., Sorokina E. I., Gieseler M., Reinecke M., Travaglio C., Hillebrandt W., Stritzinger M., 2006, A\&A, 453, 229

Chugai N. N., 1991, Soviet Astronomy Letters, 17, 210

Dahlen T., et al., 2004, ApJ, 613, 189

Dessart L., Hillier D. J., 2010, MNRAS, 405, 2141

Dessart L., Hillier D. J., 2011, MNRAS, 410, 1739

Dessart L., Livne E., Waldman R., 2010, MNRAS, 405, 2113

Dessart L., Audit E., Hillier D. J., 2015, MNRAS, 449, 4304

Falk S. W., Arnett W. D., 1977, ApJS, 33, 515

Faran T., et al., 2014a, MNRAS, 442, 844

Faran T., et al., 2014b, MNRAS, 445, 554

Grasberg E. K., Nadezhin D. K., 1976, Ap\&SS, 44, 409

Grasberg E. K., Imshenik V. S., Nadyozhin D. K., 1971, Ap\&SS, 10, 3

Kasen D., Woosley S. E., 2009, ApJ, 703, 2205

Katz B., Kushnir D., Dong S., 2013, preprint, (arXiv : 1301.6766)
Li W., Chornock R., Leaman J., Filippenko A. V., Poznanski D., Wang X., Ganeshalingam M., Mannucci F., 2011, MNRAS, 412, 1473

Li C., Hillier D. J., Dessart L., 2012, MNRAS, 426, 1671

Litvinova I. Y., Nadezhin D. K., 1985, Soviet Astronomy Letters, 11, 145 Livne E., 1993, ApJ, 412, 634

Mackey J., Bromm V., Hernquist L., 2003, ApJ, 586, 1

Mannucci F., Della Valle M., Panagia N., 2007, MNRAS, 377, 1229

Müller B., Melson T., Heger A., Janka H.-T., 2017, MNRAS, 472, 491

Nakar E., Poznanski D., Katz B., 2016, ApJ, 823, 127

Paxton B., Bildsten L., Dotter A., Herwig F., Lesaffre P., Timmes F., 2011, ApJS, 192, 3

Paxton B., et al., 2013, ApJS, 208, 4

Paxton B., et al., 2015, ApJS, 220, 15

Popov D. V., 1993, ApJ, 414, 712

Shklovskii I. S., 1960, Soviet Ast., 4, 355

Shussman T., Nakar E., Waldman R., Katz B., 2016, preprint, (arXiv: 1602.02774)

Smartt S. J., 2009, ARA\&A, 47, 63

Smith N., Li W., Filippenko A. V., Chornock R., 2011, MNRAS, 412, 1522

Sukhbold T., Ertl T., Woosley S. E., Brown J. M., Janka H.-T., 2016, ApJ, 821,38

Takáts K., et al., 2015, MNRAS, 450, 3137

Utrobin V. P., Wongwathanarat A., Janka H.-T., Müller E., 2017, ApJ, 846, 37

Valenti S., et al., 2016, MNRAS, 459, 3939

Wongwathanarat A., Müller E., Janka H.-T., 2015, A\&A, 577, A48

Young T. R., 2004, ApJ, 617, 1233

\section{APPENDIX A: THE SN EJECTA STRUCTURE AT COASTING PHASE}

Our study is focused on the light curve analysis, however, we present additionally the $\mathrm{SN}$ ejecta structure at coasting phase. This might be helpful for observers to interpret observational properties of a given $\mathrm{SN}$, like width of spectral lines of particular elements. In Figures A1, $\mathrm{A} 2, \mathrm{~A} 3$, and $\mathrm{A} 4$, we show the selected species: hydrogen $(\mathrm{H})$, helium $(\mathrm{He})$, oxygen $(\mathrm{O})$, silicon $(\mathrm{Si})$, and iron $(\mathrm{Fe})$, at day 170 for the model $\mathrm{m} 12$ with $0.045 \mathrm{M}_{\odot}$ of ${ }^{56} \mathrm{Ni}$ for all considered distributions of ${ }^{56} \mathrm{Ni}$ for this model and for all cases of explosion energy, 0.4 foe, 0.9 foe, and 1.35 foe. Similarly, Figures A6, A7, A8, A9, and A10 present ejecta structure of the model m15 with $0.056 \mathrm{M}_{\odot}$ of ${ }^{56} \mathrm{Ni}$ and considered explosion energies 0.53 foe, 1.1 foe, and 1.53 foe. Iron ("Fe") in the Figures represent a sum of mass fractions of iron-group elements included in the STELLA simulations, i.e. iron, cobalt, and nickel.

Obviously, all chemical interfaces shift forward in velocity space for higher energy. For instance, the outer boundary of ironrich material moves at $1550 \mathrm{~km} \mathrm{~s}^{-1}$ for the model $\mathrm{m} 12$ and the mixing case " $1 / 3$ " exploded with 0.4 foe (Figure A2), while it moves at $2550 \mathrm{~km} \mathrm{~s}^{-1}$ for the explosion with 1.35 foe. There is no big difference for distribution of all species except iron for different degree of uniform mixing, since we limit our study and focus on modified ${ }^{56} \mathrm{Ni}$ distribution (e.g. if compare Figures A2 and A3).

This paper has been typeset from a $\mathrm{T}_{\mathrm{E}} \mathrm{X} / \mathrm{L} \mathrm{T} \mathrm{E} \mathrm{X}$ file prepared by the author. 


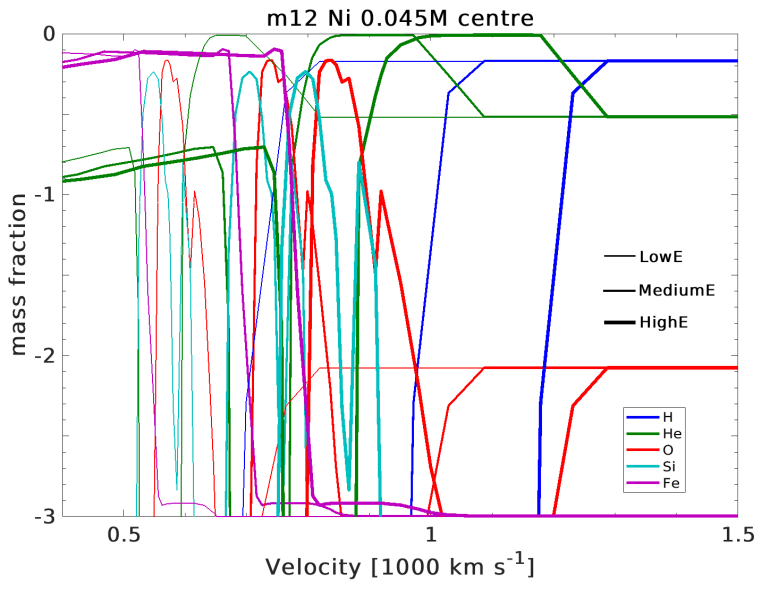

Figure A1. Selected species, hydrogen $(\mathrm{H})$, helium $(\mathrm{He})$, oxygen $(\mathrm{O})$, silicon $(\mathrm{Si})$, and iron $(\mathrm{Fe})$, at day 170 for the model $\mathrm{m} 12$ with $0.045 \mathrm{M}_{\odot}$ of ${ }^{56} \mathrm{Ni}$ distributed in the central part of the SN ejecta. "LowE" stands for low explosion energy, i.e. 0.4 foe in our study, "MediumE" stands for medium energy, i.e. 0.9 foe, and "HighE" stands for high energy, i.e. 1.35 foe.

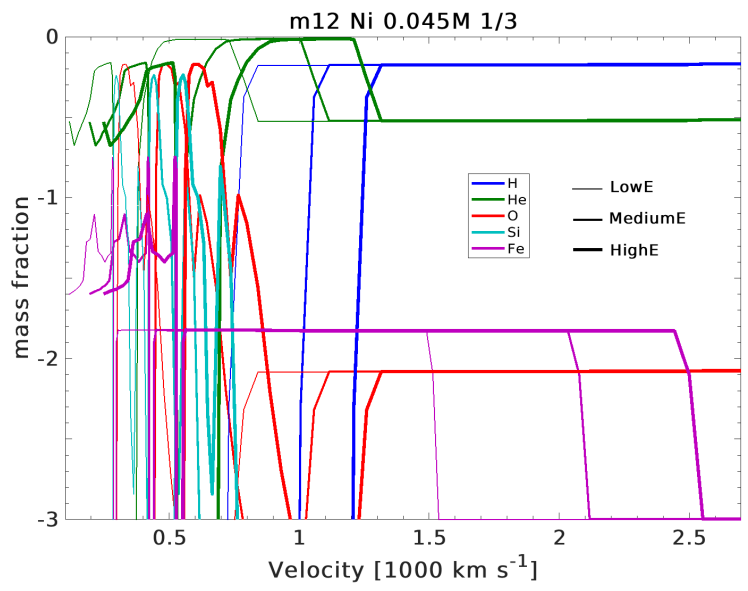

Figure A2. The same as in Figure A1 but for ${ }^{56} \mathrm{Ni}$ distributed uniformly in $1 / 3$ of the ejecta (case " $1 / 3$ ").

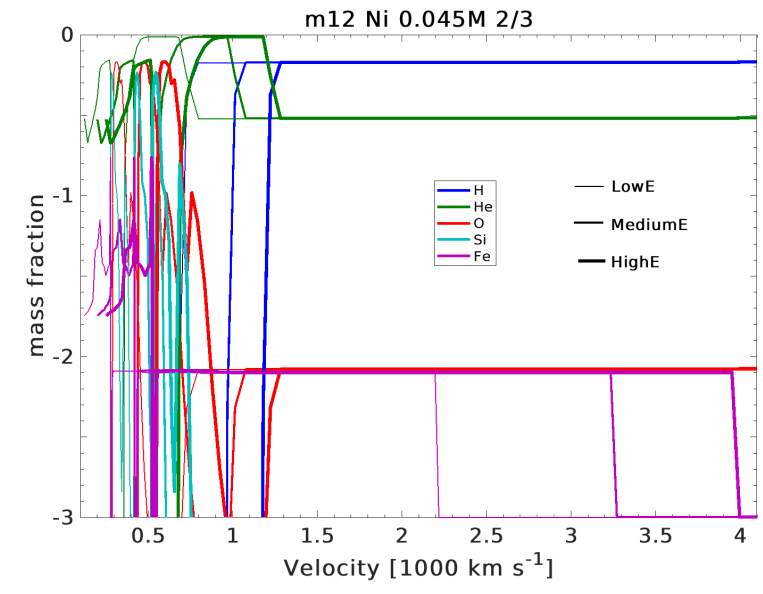

Figure A3. The same as in Figure A1 but for ${ }^{56} \mathrm{Ni}$ distributed uniformly in $2 / 3$ of the ejecta (case " $2 / 3$ ").

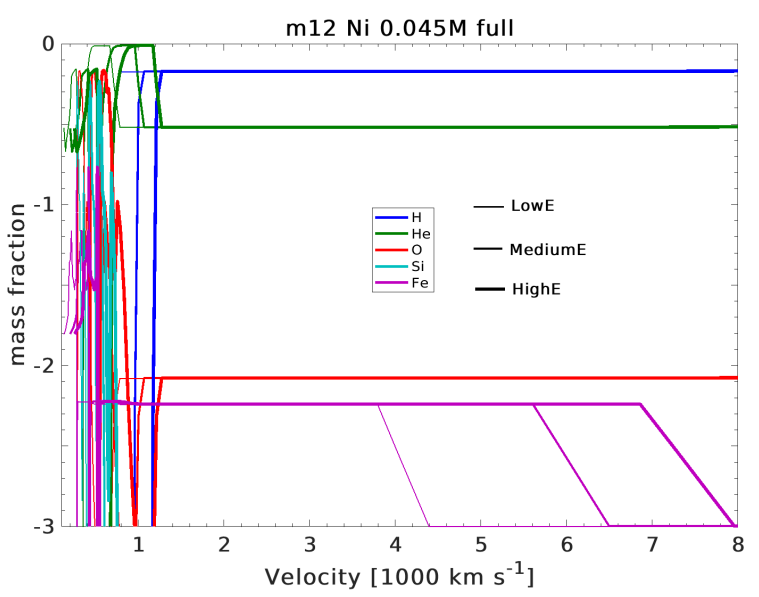

Figure A4. The same as in Figure A1 but for ${ }^{56} \mathrm{Ni}$ distributed uniformly in entire ejecta (case "full").

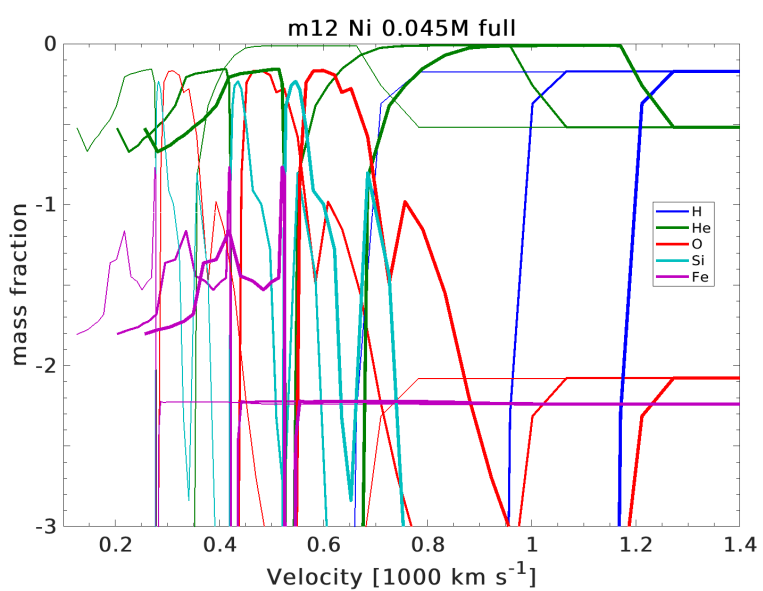

Figure A5. The same as in Figure A4 but for the inner part of the ejecta. 


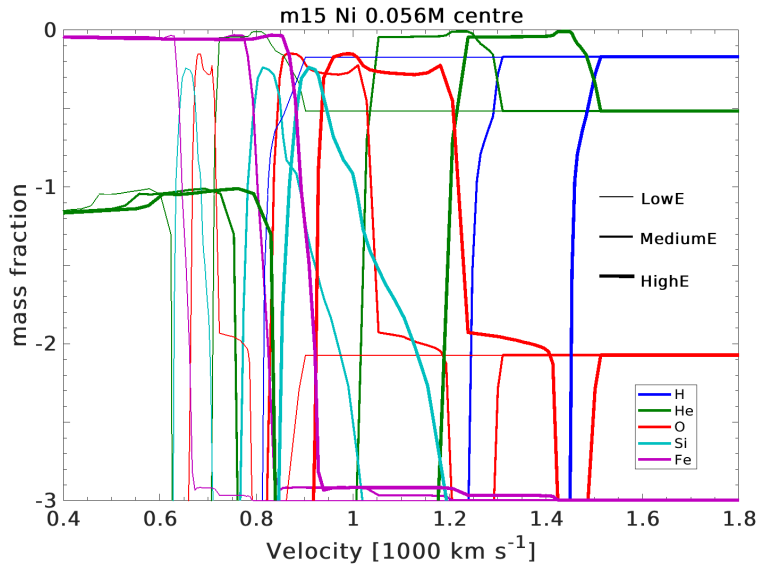

Figure A6. The same as in Figure A1 but for the model $\mathrm{m} 15$ with $0.056 \mathrm{M}_{\odot}$ of ${ }^{56} \mathrm{Ni}$ distributed in the central part of the SN ejecta. "LowE" stands for low explosion energy, i.e. 0.53 foe in our study, "MediumE" stands for medium energy, i.e. 1.1 foe, and "HighE" stands for high energy, i.e. 1.53 foe.

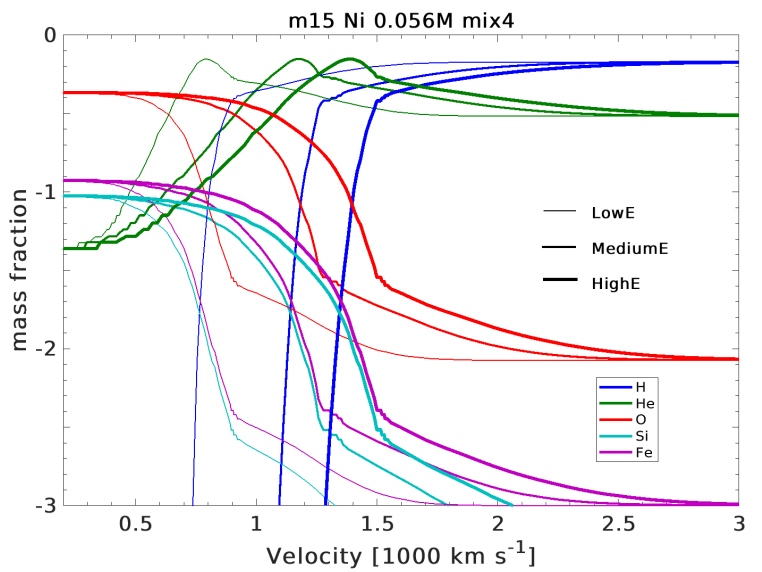

Figure A7. The same as in Figure A6 but for ${ }^{56} \mathrm{Ni}$ distributed in a boxcar manner (case "mix4").

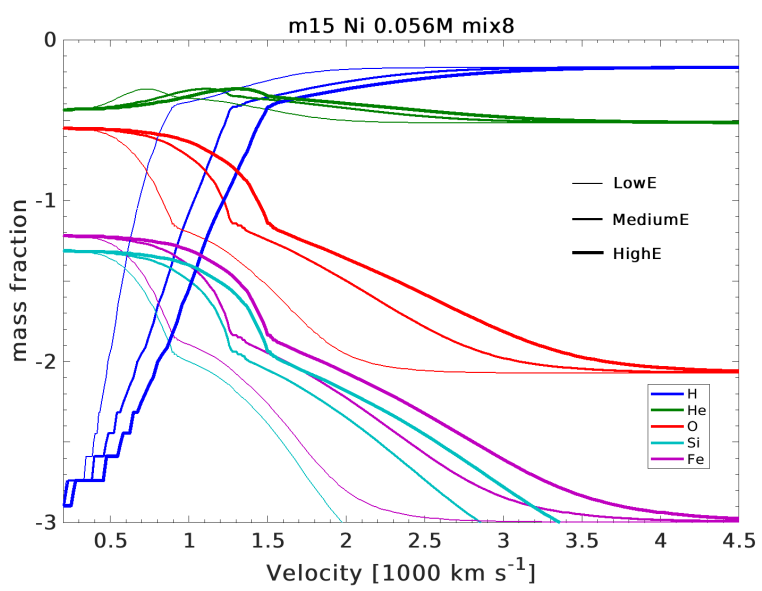

Figure A8. The same as in Figure A6 but for ${ }^{56} \mathrm{Ni}$ distributed in a boxcar manner (case "mix8").

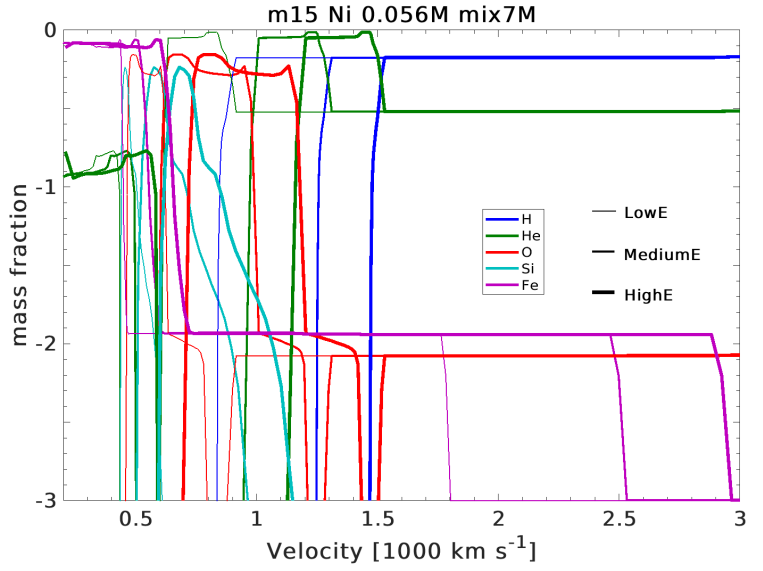

Figure A9. The same as in Figure A6 but for ${ }^{56} \mathrm{Ni}$ distributed uniformly in half of the ejecta (inner $7 \mathrm{M}_{\odot}$, case "mix7M").

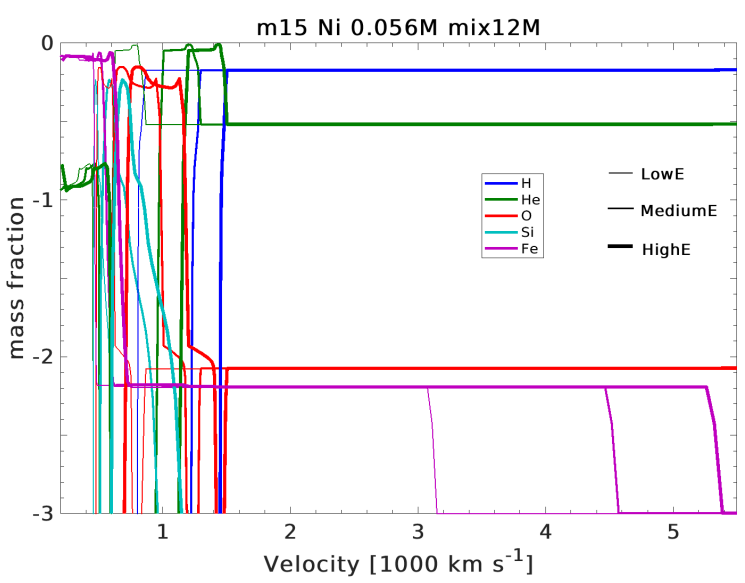

Figure A10. The same as in Figure A6 but for ${ }^{56} \mathrm{Ni}$ distributed uniformly in entire ejecta (inner $12 \mathrm{M}_{\odot}$, case "mix12M").

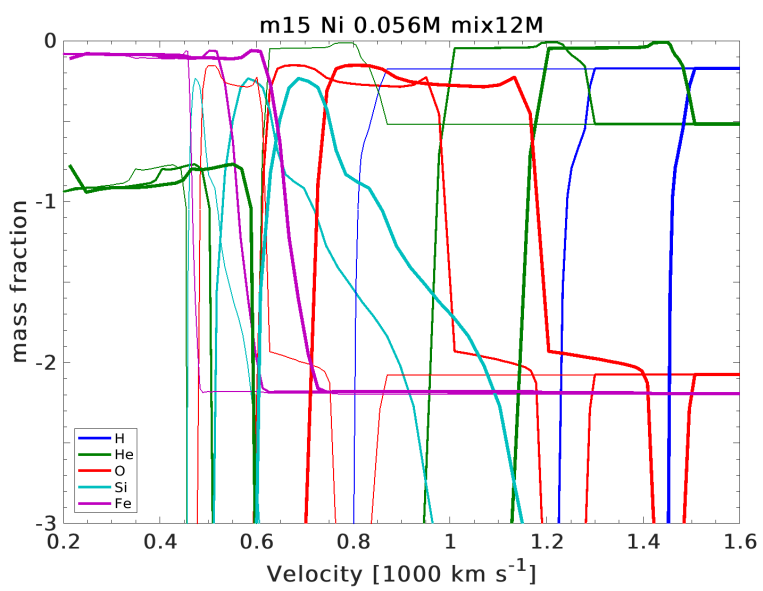

Figure A11. The same as in Figure A10 but for the inner part of the ejecta. 